\title{
DISCRETE CONFORMAL GROUPS AND MEASURABLE DYNAMICS
}

\author{
BY DENNIS SULLIVAN
}

Motivated by his study of 2nd order differential equations $a(z) w^{\prime \prime}+b(z) w^{\prime}$ $+c(z) w=0$ Poincare (1882) unveiled the vast subject of discrete subgroups of conformal transformations, $\{z \rightarrow(a z+b) /(c z+d)\}$, their associated Riemann surfaces, and the intricacies of the limit set-Cantor sets, nowhere differentiable curves, etc.

In this paper we discuss an interplay between discrete conformal groups acting on any dimensional ball $B^{d+1}$ and measurable dynamics. The development is closely related to ideas considered by Poincaré, for example,

(i) the Poincare series $\sum_{\gamma \in \Gamma}\left|\gamma^{\prime} x_{0}\right|^{s}$ where $\left|\gamma^{\prime} x\right|$ is the linear distortion of the Euclidean metric by the conformal transformation $\gamma$ and $x_{0}$ lies in the interior of $B^{d+1}$.

(ii) The interpretation of interior $B^{d+1}$ with its group of conformal transformations as the Poincaré model of non-Euclidean or hyperbolic geometry, $\mathbf{H}^{d+1}$.

The dynamics we consider take place on the $d$-sphere $=\partial B^{d+1}=$ the visual sphere at $\infty$ for $\mathbf{H}^{d+1}$. There are basically three parts to the discussion.

Part I. The ergodic properties of the geodesic flow on the associated hyperbolic manifold $\mathbf{H}^{d+1} / \Gamma$ such as (i) the excursion pattern of random geodesics into the cuspidal ends of finite volume noncompact hyperbolic manifolds.

(ii) the ergodicity of the geodesic flow relative to conformal measures and the divergence of the Poincaré series at the critical exponent $s=\delta(\Gamma)$.

Part II. The ergodic properties of $\Gamma$ acting on the tangent spaces to the Poincaré recurrent part of $S^{d}$.

Part III. An interrelation between the critical exponent $\delta(\Gamma)$ and other quantities such as (i) the Hausdorff dimension of the limit set $\Lambda \subset S^{d}$, (ii) the "square root" of the lowest eigenvalue of the Laplacian acting on $L^{2}\left(H^{d+1} / \Gamma\right)$, (iii) the entropy of the geodesic flow, (iv) general Riemannian manifolds.

We will state theorems and give ideas and references for the proofs. The discussion of Part I works for the usual Lebesgue measure on $S^{d}$ as well as for any "conformal measure" $\mu$ on $S^{d}$, that is a finite positive measure $\mu$ which satisfies $\gamma^{*} \mu=\left|\gamma^{\prime}\right|^{\delta} \mu, \gamma \in \Gamma$. These conformal measures are also important in Part III. They sometimes turn out to be Hausdorff measures, or they can be viewed as the boundary values at $\infty$ of positive eigenfunctions of

Received by the editors June 11, 1981 .

1980 Mathematics Subject Classification. Primary 32H20, 58F11, 58F17; Secondary 58F18, $10 \mathrm{~F} 05$. 
the Laplacian with the lowest possible eigenvalue, or they determine invariant measures for the geodesic flow with an interesting value for the entropy.

The discussion of Part II is restricted to Lebesgue measure or to conformal measures which share certain spatial distribution properties with Lebesgue measure. One consequence of the theory in Part II is that relative to Lebesgue measure (or any of these good conformal measures) any two ergodic components of the action on $S^{d}$ of discrete groups of conformal transformations are orbit equivalent. Another consequence is a rather complete extension and analysis of the Mostow rigidity and the Ahlfors-Bers [B] deformation theory for infinite volume groups. These results are used by Thurston [T] in his existence theory for hyperbolic structures on topological 3-manifolds.

\section{PART I}

1. Finite volume groups and diophantine approximation. We begin by recalling the classical ergodicity results for discrete groups $\Gamma$ of motions of $\mathbf{H}^{d+1}$ with finite volume fundamental domains. To these classical results we add a new result about the excursion pattern of geodesics into the cuspidal ends of a noncompact finite volume hyperbolic manifold.

The discrete group $\Gamma$ acts on the geodesic lines in $\mathbf{H}^{d+1}$ and each orbit of lines determines one geodesic in the quotient, $V=\mathbf{H}^{d+1} / \Gamma$. These geodesics fill the unit tangent bundle $T(V)$ of $V$ giving a 1-dimensional foliation whose leaves are the orbits of the geodesic flow. Thus in particular orbits of the geodesic flow for the quotient $\mathbf{H}^{d+1} / \Gamma$ are in the canonical 1-1 correspondence with $\Gamma$ orbits of unequal ordered pairs of points on the sphere at infinity $S^{d}$ of $\mathbf{H}^{d+1}$.

The geodesics may be grouped into asymptotic classes as $t \rightarrow \infty$ and as $t \rightarrow-\infty$. These classes are the leaves of two transversal foliations which intersect in the 1-dimensional geodesic foliation.

In the middle thirties, Eberhard Hopf used these asymptotic foliations to prove the geodesic flow is ergodic if $\mathbf{H}^{d+1} / \Gamma$ has finite volume. The proof also makes use of Poincaré's famous result that a finite invariant measure forces almost all geodesics to be recurrent. These foliations have been used up to the present to prove the geodesic flow is mixing, structurally stable, Bernoulli, etc. (Hedlund, Anosov, Smale, Ornstein, ....).

Returning to our discussion, one knows [T, p. 8.20] that a finite volume hyperbolic manifold $V^{d+1}=\mathbf{H}^{d+1} / \Gamma$ consists of a compact part to which cuspidal ends homeomorphic to ( $d$-torus) $\times[0, \infty)$ are attached. By the ergodicity of the geodesic flow, the random geodesic wanders continually further and further out each of these ends and returns infinitely often.

We can measure these excursions by the function dist $v(t)$, the distance from the point $v(t)$ in $V$ achieved after time $t$ to a fixed compact starting region of initial vectors $\{v\}$. It is easy to see that the event $\{v$ : dist $v(t)>T\}$ has volume comparable to $e^{-d T}$ using the volume-preserving character of the geodesic flow and the exponential decay of the size of the torus in the cuspidal end. An elementary use of the Borel-Cantelli lemma shows then for each $\varepsilon>0$ and for almost all starting directions $v$ the inequality

$$
\text { dist } v(t)<(1 / d+\varepsilon) \log (t)
$$


is eventually true. One is led to conjecture $(1 / d) \log t$ is the right upper bound function.

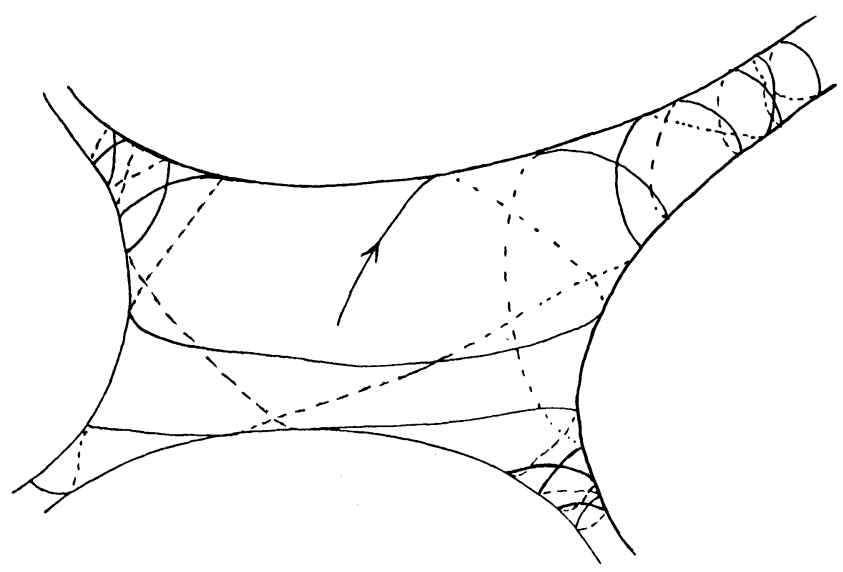

THEOREM 1. If $\Gamma$ is any cofinite volume, but not cocompact, discrete group of non-euclidean motions in $\mathbf{H}^{d+1}$, then for almost all starting directions $v$ in $\mathbf{H}^{d+1} / \Gamma$

$$
\limsup _{t \rightarrow \infty} \frac{\operatorname{dist} v(t)}{\log t}=\frac{1}{d}
$$

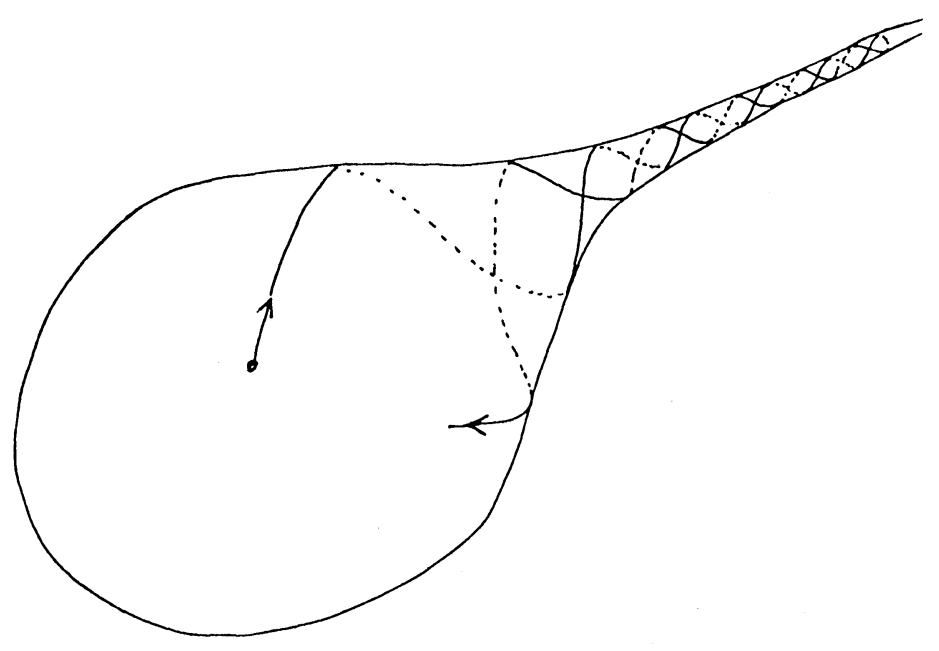

FIGURE 1 
This theorem is proved in [S(i)] using a proposition about disjoint spheres resting on a Euclidean hyperplane implying an associated sequence of sets in the hyperplane are sufficiently independent in the sense of probability to have the full Borel-Cantelli lemma-divergence of the sum of measures implies the infinitely often event has positive measure.

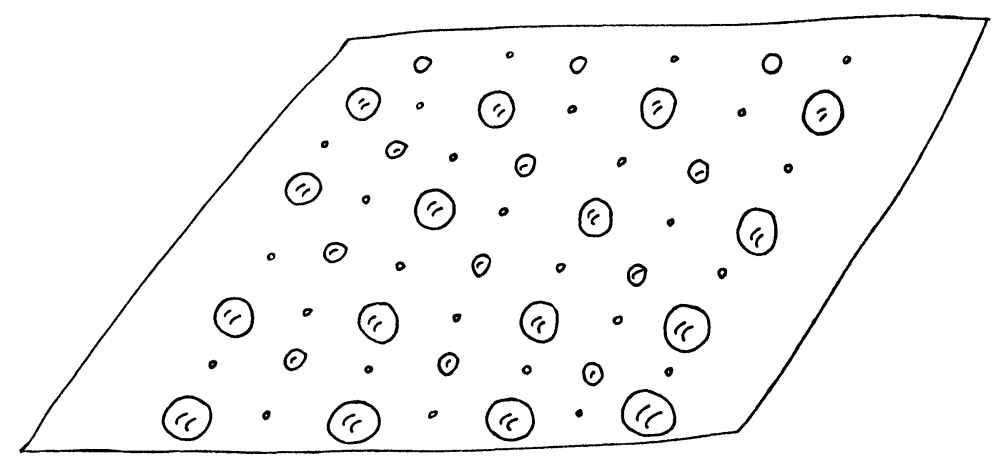

FIGURE 2

To use this proposition there must be enough spheres of each size. In the proof, this abundance of spheres comes from the mixing property of the geodesic flow.

The problem of geodesic excursion turns out to be equivalent (an oral communication of David Kahzdan) to the problem of approximating a general point on the sphere at infinity by the orbit of a parabolic cusp. Thus the proof above yields a discrete group-ergodic proof of Khintchine's metric theory of diophantine approximation. For this application of Theorem 1 we take $\Gamma$ to be $P S l(2, Z)$.

If we consider the analogous approximation discussion for complex numbers and imaginary quadratic fields $Q(\sqrt{-d})$ and let $\Gamma$ run through the Bianchi groups $\operatorname{PSl}(2, \vartheta \sqrt{-d})$ we obtain just as easily a Khintchine theorem in this context. The statement proved in [S(i)] follows.

Let $0<a(x) \leqslant 1$ be a function so that the value up to bounded ratio only depends on the argument up to bounded ratio. For the positive integer $d$ denote by $\vartheta$ the ring of integers in $Q \sqrt{-d}$.

THEOREM 2. For almost all complex numbers $z$ there are infinitely many pairs $p, q \in \vartheta \times \vartheta$ so that $\operatorname{ideal}(p, q)=\vartheta$ and $|z-p / q|<a(|q|) /|q|^{2}$ iff $\int^{\infty}\left(a(x)^{2} / x\right) d x=\infty$.

For the Gaussian field, LeVeque (1952) proved a variant of this along the lines of the Khintchine real result.

2. Groups with infinite volume fundamental domains. As suggested already, E. Hopf's foliation proof of ergodicity of the geodesic flow is based on the Poincare recurrence of the geodesic flow which follows in the finite volume case from the Poincaré recurrence theorem. In fact, E. Hopf proved (1939) 
the geodesic flow on an infinite volume hyperbolic manifold was either

(i) completely nonrecurrent. Namely, the random geodesic eventually leaves very compact set forever, or

(ii) the geodesic flow is completely recurrent and ergodic.

Hopf asked what geometric conditions might separate these two cases. In [S(ii)] we studied the ergodicity question independently of Hopf's 1939 paper. We found the same dichotomy for the geodesic flow, namely (i) completely nonrecurrent of (ii) ergodic by showing this dichotomy agrees with a dichotomy which exists for any Riemannian manifold [S(ii), §2]. Namely, random motion on a Riemannian manifold is either transient or recurrent.

In classical function theory this dichotomy for a Riemannian surface can be expressed in terms of the existence or nonexistence of a Green's function, a positive function with the standard Green's singularity at a point and harmonic outside the point.

Poincare himself (1906) analyzed this dichotomy using the fuchsian group representation of a Riemann surface and reformulated the condition in terms of the convergence or divergence of a series (now called the Poincaré series),

$$
\sum_{\gamma \in \Gamma}\left|\gamma^{\prime}\left(z_{0}\right)\right|<\infty \text { or not. }
$$

Here $\Gamma$ is a discrete group of conformal transformations of $\{z:|z|<1\}$, $\left|z_{0}\right|<1$, and $\left|\gamma^{\prime}(z)\right|$ is the linear distortion of the Euclidean metric.

The Poincaré dichotomy in terms of the series makes sense for any discrete group of conformal transformations of the Euclidean ball $B^{d+1}$. The dichotomy becomes

$$
\sum_{\gamma \in \Gamma}\left|\gamma^{\prime}\left(x_{0}\right)\right|^{d}<\infty \text { or not. }
$$

THEOREM 3. The dichotomy, $\Sigma_{\Gamma}\left|\gamma^{\prime}\left(x_{0}\right)\right|^{d}<\infty$ or not, is equivalent to the Hopf dichotomy, the complete nonrecurrence or the ergodicity of the geodesic flow for $\mathbf{H}^{d+1} / \Gamma$.

The proof in [S(ii)] makes use of random walks and was motivated by a proposition in Garnett [G] about the measurable functions on a compact foliated manifold harmonic in each leaf being constant on almost all leaves.

Thus if the series $\Sigma_{\gamma \in \Gamma}\left|\gamma^{\prime}\left(x_{0}\right)\right|^{d}$ converges we have a completely noncurrent geodesic flow relative to Lebesgue measure on $\mathbf{H}^{d+1} / \Gamma$. One may try to restore ergodicity by replacing Lebesgue measure by a different geometrically meaningful measure.

To this purpose consider again the Poincaré series $\Sigma_{\gamma \in \Gamma}\left|\gamma^{\prime}\left(x_{0}\right)\right|^{s}$ with an exponent $s$. One defines the critical exponent $\delta(\Gamma)$ so that the Poincare series converges for $s>\delta(\Gamma)$ and diverges for $s<\delta(\Gamma)$. In [S(iii)] which generalized Patterson [P] it was shown there is a probability measure $\mu$ on the limit set $\Lambda(\Gamma) \subset S^{d}$ so that $\gamma^{*} \mu=\left|\gamma^{\prime}\right|^{\delta} \mu, \gamma \in \Gamma$, where $\delta=\delta(\Gamma)$. Moreover, $\delta(\Gamma)$ is the minimum power for which such a measure exists. [S(iii), § 2].

Just as Lebesgue measure is associated to an invariant measure for the geodesic flow, such conformal measures $\mu$ determine invariant measures for 
the geodesic flow [S(iii), \$4]. Basically, the formula $\gamma^{*} \mu=\left|\gamma^{\prime}\right|^{\delta} \mu$ and the formula $|\gamma x-\gamma y|^{2}=\left|\gamma^{\prime} x\right|\left|\gamma^{\prime} y\right||x-y|^{2}$ implies $\mu \times \mu /|x-y|^{2 \delta}$ is a $\Gamma$-invariant measure on $S^{d} \times S^{d}$. This invariant measure is combined with arc length to yield a natural invariant measure for the geodesic flow of $\mathbf{H}^{d+1} / \Gamma$.

Theorem 3 above has the following generalization.

THEOREM 4. The geodesic flow is ergodic relative to a conformal measure $\mu$ of dimension $\delta$ iff the Poincaré series $\Sigma_{\gamma \in \Gamma}\left|\gamma^{\prime} x_{0}\right|^{\delta}$ diverges. In this divergence case the conformal measure $\mu$ satisfying $\gamma^{*} \mu=\left|\gamma^{\prime}\right|^{\delta} \mu, \gamma \in \Gamma$, is unique.

It is interesting for what follows to describe the evolution of the proof of Theorem 4. We have mentioned the proof of Theorem 3 using a random walk along the leaves of the asymptotic foliation in [S(ii), \$2] motivated by [G]. The idea behind that proof was simply that a random path in hyperbolic space hits a definite point at $\infty$. Thus there is a $\Gamma$-invariant map of bi-infinite random paths to geodesics by looking at the endpoints. Recurrence of Brownian motion implies the shift map on bi-infinite paths of $\mathbf{H}^{d+1} / \Gamma$ is ergodic and thus so also is its quotient, the geodesic flow.
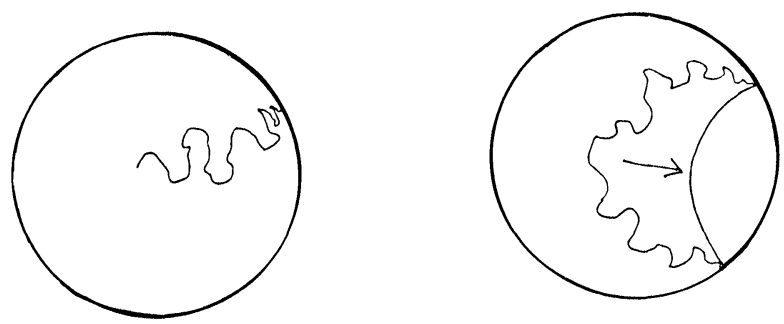

This proof of Theorem 3 was described in [S(iii), §7] together with an extension to those cases of Theorem 4 where $\delta>d / 2$. For the extension a conditioned Markov process with transition probabilities

$$
e^{-\lambda t} \phi(y) p_{t}(x, y) / \phi(x)
$$

was considered. Here $\lambda=\delta(\delta-d), \phi$ is a positive $\lambda$-eigenfunction of the Laplacian with boundary values at infinity the conformal measure $\mu$, and $p_{t}(x, y)$ is the transition probability for the usual Brownian motion on $\mathbf{H}^{d+1} / \Gamma$. (See Part III for more discussion of these ideas.)

This Markov process proof does not work for $\delta<d / 2$. Recently, a more elementary proof of the case $\delta=d$ was found (related to joint work [AS] with Jon Aaronson) which succeeds just as well for all $\delta$. First, E. Hopf's proof that there is a dichotomy between complete nonrecurrence and ergodicity works for any conformal measure $\mu$ [S(iii), §4]. Second, recurrence can be checked by an abstract Borel-Cantelli lemma analogous to the one used in $\S 1$. This step requires an elementary geometric inequality which says the probability that a geodesic starting in a ball $B$ returns to $B$ at a later time $t$ and at an even time $t+s$ is at most a universal constant times the product of the respective probabilities the geodesic returns respectively at times $t$ and time $s$. 
This inequality for the measure $\mu$ uses the transformation formula $\gamma^{*} \mu=$ $\left|\gamma^{\prime}\right|^{\delta} \mu$ to see that the $\mu$ shadow of a ball $\gamma B$ as viewed from $B$ is on the order of $e^{-\delta x}$ where $x$ is the hyperbolic distance from $B$ to $\gamma B$.

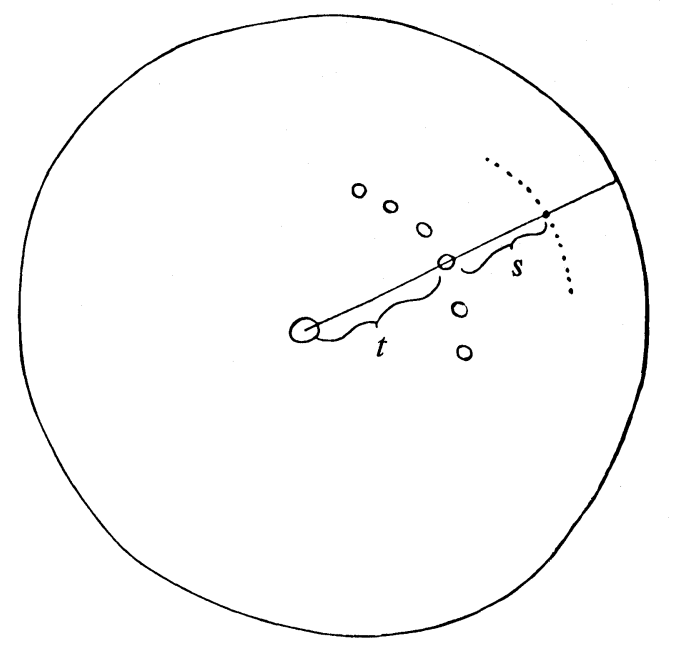

This Markov type inequality has another application in the context of Lebesgue measure, to yield a sharper form of ergodicity when the series $\Sigma\left|\gamma^{\prime} x_{0}\right|^{d}$ diverges. This sharper ergodicity is rational ergodicity as explained in [A]. It implies in particular that the sojourn times in $B$ up to time $T$ have a pattern of divergence which is just that of the diverging Poincare series [AS]. Up to now it is a problem to derive the rational ergodicity for conformal measures of dimension $\delta$ and thus the relation between sojourn times up to time $T$ and the rate of divergence of $\Sigma\left|\gamma^{\prime} x_{0}\right|^{\delta}$.

3. There is a relation between this ergodicity of geodesics or equivalently ergodicity of $\Gamma$ acting on pairs in $S^{d}$ and a purely measurable form of Mostow rigidity. Suppose $\Phi$ is a Borel map: $S^{d} \rightarrow S^{d}$ conjugating one action of a discrete conformal group $\Gamma_{1}$ to that of another $\Gamma_{2}$. Suppose $\Phi$ is nonsingular with respect to conformal measures $\mu_{1}$ and $\mu_{2}$ of dimension $\delta$. That is, $\gamma^{*} \mu_{1}=\left|\gamma^{\prime}\right|^{\delta} \mu_{1}$ for $\gamma \in \Gamma_{1}, \gamma^{*} \mu_{2}=\left|\gamma^{\prime}\right|^{\delta} \mu_{2}$ for $\gamma \in \Gamma_{2}$ and $\mu_{1}(A)>0$ iff $\mu_{2}(\Phi A)>0$. Then

TheOReM 5. If the Poincaré series for $\Gamma_{1}$ diverges at $\delta$, the map $\Phi$ agrees $\bmod \mu_{1}$ with a conformal conjugacy between $\Gamma_{1}$ and $\Gamma_{2}$.

Proof. Since the Poincaré series for $\Gamma_{1}$ diverges at $\delta$, the measure $\mu_{1} \times \mu_{1} /{ }_{\mid} x-\left.y\right|^{2 \delta}$ is ergodic for $\Gamma_{1}$ acting on $S^{d} \times S^{d}$ (Theorem 4). Since $\Phi$ is nonsingular, $\Phi \times \Phi$ is a measurable conjugacy between the two product actions on $S^{d} \times S^{d}$. Thus $\Gamma_{2}$ acts ergodically relative to $\mu_{2} \times \mu_{2} /|x-y|^{2 \delta}$ and $\Phi \times \Phi$ carries $\mu_{1} \times \mu_{1} /|x-y|^{2 \delta}$ to an invariant measure for $\Gamma_{2}$ which must be a constant times $\mu_{2} \times \mu_{2} /|x-y|^{2 \delta}$. It follows immediately for $\mu_{1}$ almost all pairs $x, y$ that $|\Phi x-\Phi y| /|x-y|$ is a product of a function of $x$ 
and one of $y$. Thus $\Phi$ preserves cross ratios

$$
|x-y| \cdot|z-w| /|x-w| \cdot|y-z| \text { for } \mu_{1} \text { almost all four-tuples. }
$$

Renormalizing $\Phi$ so that for a generic $w, w$ and $\Phi(w)$ are at infinity and working in $R^{d}$, the new $\Phi$ preserves $|x-y| /|y-z|$ for $\mu_{1}$ almost all triples. Cyclically permuting $x, y, z$ shows $\Phi$ now takes $\mu_{1}$ almost all triangles to similar triangles. Renormalizing further by a similarity $\Phi$ preserves one triangle. Now it follows $\Phi$ preserves distances for almost all pairs. Thus $\Phi$ agrees $\bmod \mu_{1}$ with an isometry. Returning to the original situation we have a conformal conjugacy between $\Gamma_{1}$ and $\Gamma_{2}$. This proves Theorem 5 .

Application of Part I. For a compact Riemann surface $S$ of genus $g$ there is a $6 g-6$ parameter (Teichmüller) space of discrete groups $\Gamma$ acting on $\mathbf{H}^{2}$ so that $\mathbf{H}^{2} / \Gamma$ is homeomorphic to $S$. All these groups act ergodically on $S^{1} \times S^{1}$ so Theorem 6 implies

COROLlaRy 1. The $6 g-6$ parameter space injects into the abstract ergodic theory of actions of $\Gamma=\pi_{1} S$ on the circle. If two of these $\pi_{1} S$ actions on $S^{1}$ are measurably conjugate (preserving sets of positive measure) the corresponding Riemann surfaces are conformally equivalent.

Note. In this compact surface case a very different proof by Marina Ratner [Ra] has shown a surprising related result-if two classical horocycle flows are measure-theoretically conjugate the underlying Riemann surfaces are homeomorphic, even conformally isomorphic.

For general geometrically finite groups Theorem 5 says the conformal type is determined by the critical exponent (which is also Hausdorff dimension of the limit set, see Part III) and the measure-theoretic conjugacy class of the $\Gamma$ action on the limit set relative to the unique conformal measure.

A more refined analysis by Tukia [Tu] has shown that in the geometrically finite case without cusps the critical exponent is even determined by the measure-theoretical conjugacy of the action of $\Gamma$ on $S^{d}$ relative to the conformal measure (which in this case is the Hausdorff measure [S(iii), §3]) at least when the conjugacy is continuous.

\section{PART II}

Now we discuss the ergodic properties of the action of a discrete conformal group on the tangent spaces to $S^{d}$. Say that $\lambda>0$ belongs to the ratio set of a group action with a quasi-invariant measure $\mu$ if for each set $B$ of positive measure and each $\varepsilon>0$ there are infinitely many groups elements $\gamma \in \Gamma$ so that $\gamma$ carries a positive part of $B$ back into $B$ with Radon-Nikodym derivative $D \gamma(x)$ within $\varepsilon$ of $\lambda$. The ratio set is independent of the choice of $\mu$ in the measure class of $\mu$.

The concept of ratio set is only interesting in the recurrent part of the action. The recurrent part is by definition the complement of the wandering part. The wandering part is by definition the unique maximal subset (mod null sets) where the action has a fundamental set-one which cuts each orbit in one point. 
THEOREM 6. For the recurrent part in $S^{d}$ of the action of a discrete conformal group (relative to Lebesgue measure) the ratio set consists of all positive real numbers.

There are two ingredients in the proof. The first one is an abstract proposition in ergodic theory due to Krieger and K. Schmidt.

Proposition. For any recurrent group action the number 1 belongs to the ratio set.

The proof of the proposition may be found in Klaus Schmidt [Sc].

This proposition has a strong consequence for any discrete conformal group on its recurrent part relative to Lebesgue measure where of course $D \gamma(x)=\left|\gamma^{\prime}(x)\right|^{d}$, or relative to any conformal measure $\mu$ which by definition satisfies $D \gamma(x)=\left|\gamma^{\prime} x\right|^{\delta}$.

Namely, working in $R^{d}$ instead of $S^{d}$, we can come back to any recurrent set of positive measure $B$ in $R^{d}$ by an infinite sequence of elements $\gamma_{1}$, $\gamma_{2}, \ldots$ which carry the annular regions near the isometric circles from $B$ back into $B$.

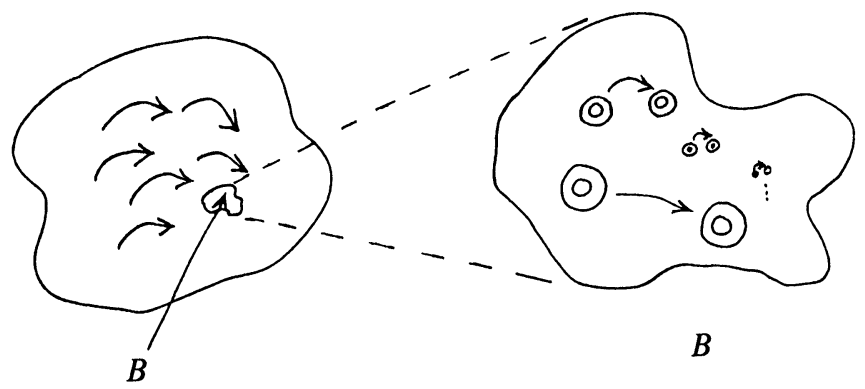

To see this we apply the proposition and recall that a conformal transformation of $R^{d}$ is the composition of an inversion followed by a Euclidean isometry.

Now $\left|\gamma^{\prime} x\right|$ is just $(a / r)^{2}$ (coming for the inversion factor) where $a$ is the radius of the isometric sphere (= inversion sphere) and $r$ is the distance of $x$ from the center. So to see $\lambda$ is in the ratio set consider the annulus between the isometric sphere and the sphere where $(a / r)^{2}=\lambda$. These annuli have a definite shape and the diameters are arbitrarily small because the group is discrete and we have infinitely many elements $\gamma$ so that $\left|B \cap \gamma^{-1} B\right|>0$.

We can apply Lebesgue density to find a small enough size for which such annuli intersecting $B^{\prime} \subset B$ are filled by points of $B$ to a very high percentage. We can be sure most of these points are carried back to $B$ if at least one is carried to $B^{\prime}$ since the derivative only varies in a fixed proportion on these annuli. By this argumentation we find $\lambda$ approximately anywhere we look. For more details see [S(ii), §1].

Now we state a theorem about the action of $\Gamma$ on tangent spaces to $S^{d}$. 
THEOREM 7. In the recurrent part of the action of a discrete conformal group relative to Lebesgue measure the action on tangent spaces to $S^{d}$ is irreduciblethere is no measurable field of $\Gamma$ invariant subspaces.

The proof of Theorem 7 begins with the analysis of Theorem 6. Now however instead of moving concentrically from the isometric sphere to find the desired scale distortion of the derivative we move around the isometric sphere to find enough twisting to destroy the invariance of any measurable field of subspaces.

In fact, we look at a set of positive measure where the field is nearly parallel. Then we recur in that set with $\gamma$ 's having small isometric spheres so Lebesgue density applies. The Euclidean isometry factor preserves the parallel property and the inversion destroys it. Thus $\gamma$ the product cannot preserve it. For more details see [S(ii), §1, §7].

REMARK. The properties of Lebesgue measure used in the above proofs are

(i) the transformation law $\gamma^{*} \mu=\left|\gamma^{\prime}\right|^{d} \mu$.

(ii) Lebesgue measure on $R^{d}$ is not unfairly concentrated near any hypersphere or near any ray.

Now any conformal measure satisfies (i) (with a different power) by definition so this part of the proof is valid. Sometimes one can verify the gemetric property (ii) for particular conformal measures. In those cases Theorems 6 and 7 are then also valid for the recurrent part of a conformal measure.

THEOREM. For any finitely generated discrete conformal group on $S^{2}$ we have that any nonatomic conformal measure on the limit set (assumed to be a proper subset of $S^{2}$ ) is recurrent.

(This is proved by the same Ahlfors-Borel series argument which shows the finiteness of cusps [S(vi), Part I]. One has in fact $\Sigma_{\Gamma}\left|\gamma^{\prime} z\right|^{N}<\infty$ for only finitely many orbits in the limit set. So any nonatomic conformal measure on the limit set is recurrent.)

Now for certain limits of geometrically finite groups-the hyperbolic half cylinder of $[\mathbf{S}(\mathbf{v})]$ - one knows enough about the canonical conformal measure to verify (ii). Thus for these conformal measures Theorems 6 and 7 are valid.

Applications of Part II. (1) Theorem 7 implies a generalization of the (quasi-conformal $\rightarrow$ conformal) part of the Mostow rigidity theory.

COROllary 2. Suppose $\phi: S^{d} \rightarrow S^{d}$ is a quasi-conformal homeomorphism $(d \geqslant 2)$ conjugating one discrete conformal group $\Gamma$ to another $\Gamma^{\prime}(\gamma \in \Gamma$ iff $\left.\phi \gamma \phi^{-1} \in \Gamma^{\prime}\right)$. Further suppose the derivative of $\phi$ is a similarity transformation at almost all points of the nonrecurrent part of the action of $\Gamma$. Then $\phi$ is a conformal transformation.

SKETCH OF PROOF. If not, the conformal distortion of $\phi$ on the recurrent part is expressed (in particular) by a $\Gamma$ invariant measurable family of proper subspaces. Such a family is impossible by Theorem 7. Thus at almost all points the derivative of $\phi$ is a similarity transformation. Thus by a standard fact in quasi-conformal mappings $\phi$ is actually conformal. For more details see [S(ii), §7]. 
(2) Say two groups actions are orbit equivalent relative to quasi-invariant measures if (after discarding invariant sets of zero measure) there is a Borel bijection preserving orbits and sets of positive measure.

Also recall any recurrent group action relative to a quasi-invariant measure can be decomposed into a measurable family of ergodic actions. The base of this family is a separable Lebesgue space consisting of a continuous part (either vacuous or isomorphic to $(0,1)$ ) and a countable set of atoms.

So the ergodic decompositions plus the wandering part can be labeled by an element in

$$
\{0,1 / 2,1\} \times\{0,1\} \times\{0,1,2, \ldots, \omega\}
$$

which we call the signature of the action. The $\{0,1 / 2,1\}$ factor tells whether the wandering part has zero, partial, or full measure, the second factor tells whether $(0,1)$ is present or not, the third factor counts the atoms.

THEOREM 8. (i) For any discrete group $\Gamma$ of conformal transformations of $S^{d}$ there is countable partition of $S^{d}=X_{1} \cup X_{2} \cup \cdots$ and elements $\gamma_{1}, \gamma_{2}, \ldots$ in $\Gamma$ so that

$$
\psi=\gamma_{1} / X_{1} \cup \gamma_{2} / X_{2} \cup \cdots
$$

is a measurable bijection of $S^{d}$ whose orbits are precisely the orbits of $\Gamma$ (modulo the null sets of any quasi-invariant measure).

(ii) Any two discrete groups of conformal transformations (possibly in different dimensions) with the same signatures relative to Lebesgue measure are orbit equivalent.

SKeTCH OF PROOF. (i) Given $\xi$ in $S^{d}$ choose an upper half space model $R_{+}^{d+1}$ of $\mathbf{H}^{d+1}$ with $\xi$ at $\infty$. A fixed reference orbit in $\mathbf{H}^{d+1}$ now determines a discrete set in $R_{+}^{d+1}$. If we move to $\xi^{\prime}$ on the same $\Gamma$ orbit as $\xi$, the discrete set is only changed by similarity. We can view this situation as saying the orbit in $S^{d}$ of a discrete conformal group has the natural structure of a horizontally and vertically discrete set in $R_{+}^{d+1}$ well defined up to similarity.

This structure implies the orbits of $\Gamma$ on $S^{d}$ can be assigned a $Z$-ordering. An abstract formal proof goes as follows. This structure implies (i) the equivalence relation defined by $\Gamma$ orbits is amenable, bounded measurable functions of pairs of points in $\Gamma$ orbits can be averaged in a $\Gamma$-invariant way (use the discreteness property above and the fact that the group of similarities is amenable to form the average). (ii) Now a new general result of Connes, Feldman, Ornstein, and Weiss [C et al] says the orbits can be Z-ordered.

(ii) Kreiger [K] has classified actions of $Z$ up to orbit equivalence by the patterns of volume distortion as expressed by the Radon-Nikodym derivative. We use only a part of this which says that if the ratio set contains all values as indicated by Theorem 6 , then the orbit equivalence class is determined uniquely (and it is called the unique $\mathrm{III}_{1}$ ).

Thus since any discrete conformal group is orbit equivalent to an action of $Z$ by (i) it is also orbit equivalent to an integral of $\mathrm{III}_{1}$ 's over its ergodic decomposition (on the recurrent part relative to Lebesgue measure). 


\section{PART III}

Now we discuss the interrelationships between critical exponent, Hausdorff dimension, the spectrum of the hyperbolic Laplacian, entropy and general Riemannian manifolds.

Poincare in his long paper on Kleinian groups (1883) observed the following-if $\Gamma$ is a discrete conformal group acting on $S^{2}$ obtained by a general perturbation of a group $\Gamma_{0}$ whose limit set was a round circle, then the limit set of the perturbed $\Gamma$ could be a very wiggly curve without a tangent at a dense set of points.
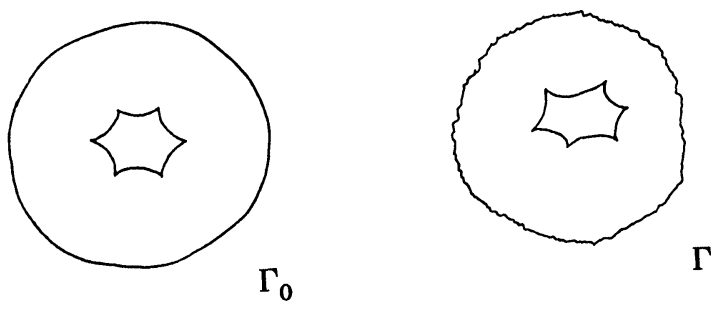

Rufus Bowen (1978) proved first that such wiggly limit curves were nonrectifiable and then that their Hausdorff dimension was greater than one. The nonrectifiability can be seen using Theorem 5 and the Riemann mapping theorem.

Namely, by classical function theory the Riemann map between the round disk and the domain of discontinuity is absolutely continuous on the boundary if the curve is rectifiable (Bowen's first step). By Theorem 5 with respect to 1-dimensional Hausdorff measure the boundary map would be part of a conformal conjugacy between $\Gamma_{0}$ and $\Gamma$. This argument gives a different proof of a general form of Bowen's nonrectifiability theorem.

THEOREM 9. Let $\Gamma$ be a discrete conformal group preserving a simply connected Jordan domain. Suppose for the corresponding group $\Gamma_{0}$ in the disk the Poincare series diverges at $s=1$. Then the boundary of the Jordan domain is either nonrectifiable or a round circle.

The second part of Bowen's Hausdorff dimension proof was to construct, using Markov partitions on the wiggly curve, a finite measure and a real number $\delta$ so that the amount of mass in the disk of radius $r$ about any point on the curve is comparable to $r^{\delta}$. It follows from the definition that $\delta$ is the Hausdorff dimension and the Hausdorff measure in dimension $\delta$ is finite and positive. (Bowen then concludes the argument by the reasoning-if the Jordan curve is not a circle it is nonrectifiable and thus $\delta>1$.)

In [S(iii)], this situation concerning Hausdorff measure was extended to all geometrically finite group without cusps (there called convex cocompact groups). The new ingredient difference from Bowen's discussion is a construction generalizing one in Patterson's paper [P] which produces a finite conformal measure $\mu$ on the limit set of any discrete conformal group $\Gamma$. Here $\gamma^{*} \mu=\left|\gamma^{\prime}\right|^{\delta} \mu$ for $\gamma \in \Gamma$ and $\delta$ is the critical exponent of the Poincare series $\left(\Sigma_{\Gamma}\left|\gamma^{\prime} x_{0}\right|^{s}\right.$ converges for $s>\delta$ and diverges for $\left.s<\delta\right)$. 
Then rather simple and general arguments [S(iii), §2, §3, §7] show

TheOREM 10. For a convex-compact group $\Gamma$ in any dimension (that is geometrically finite without cusps) the Hausdorff dimension of the limit set is the critical exponent $\delta$. The Hausdorff $\delta$-measure $\mu$ is finite and positive. In fact $\mu$ (disk of radius $r$ centered on a limit point) is comparable to $r^{\delta}$. The group $\Gamma$ acts ergodically on $\mu \times \mu$ and the Poincaré series diverges at $\delta$.

If $p$ belongs to $B^{d+1}$, let $\Phi(p)$ be the $\delta$ Hausdorff measure of the limit set in the visual metric on rays from $p$. Then $\Phi$ is $\Gamma$-invariant, it is an eigenfunction of the Laplacian with eigenvalue $\delta(\delta-d)$. If $\delta>d / 2$; $\Phi$ is square integrable in $\mathbf{H}^{d+1} / \Gamma$.

Tukia [Tu] has used this theorem to show that if two such actions on the limit set are continuously and absolutely continuously conjugate with respect to Hausdorff measure, the groups are conformally conjugate. (Note the comparison with Theorem 5.)

One consequence is the following analogue of Bowen's Hausdorff dimension result for discrete conformal groups in the 3-sphere. Start with a compact hyperbolic 3-manifold $V$ which contains a totally geodesic embedded surface (e.g. use the units of the quadratic form $x_{0}^{2}+x_{1}^{2}+x_{2}^{2}-\sqrt{2} x_{3}^{2}$ and set $\left.x_{0}=0\right)$.

Consider the universal cover of $V$ as a hyperplane $\mathbf{H}^{3}$ contained in $\mathbf{H}^{4}$. Now following Thurston's ideas for $\mathbf{H}^{2} \subset \mathbf{H}^{3}$ bend the hyperplane $\mathbf{H}^{3} \subset \mathbf{H}^{4}$ equivariantly along the inverse image of the geodesic surface in $V$. This bends the group and constructs a quasi-conformal deformation of $\Gamma=\pi_{1} V^{3}$ thought of as a discrete group acting on $\mathbf{H}^{4}$.

The limit set is a topological two-sphere from which infinitely many hemispheres have protruded (the surface analogue of Thurston's "Mickey Mouse" example [T]).

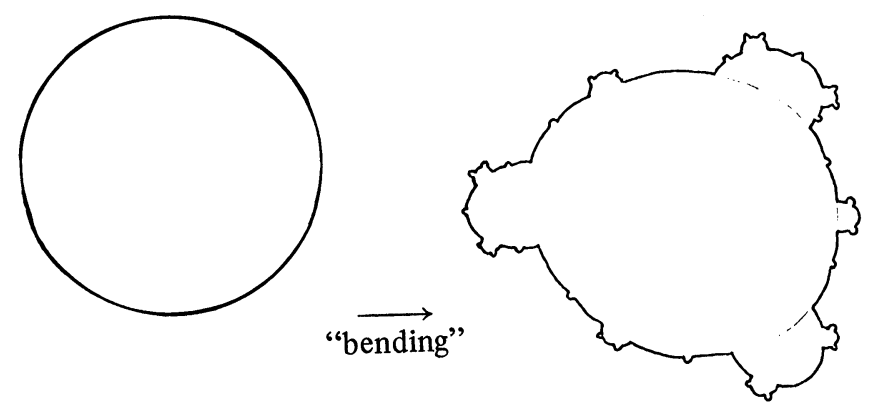

The topological 2-sphere is the boundary of a quasi-conformal 3-ball by construction. Its Hausdorff dimension must be strictly greater than two using Theorems 5 and 10, and the structure of sets of finite Hausdorff 2-measure (Federer).

We have tried to develop the results of Theorem 10 for more general groups and the attempt is mostly successful for general geometrically finite groups with cusps. Some parts of the discussion work for general groups and these even have interpretations for general Riemannian manifolds. 
As an indication of this development, we list some results for discrete conformal groups, the Poincaré series $\Sigma\left|\gamma^{\prime} x_{0}\right|^{s}$, the critical exponent $\delta(\Gamma)$, conformal measures, entropy, etc.

(1) As mentioned above for $\delta=\delta(\Gamma)$ there is always a finite positive conformal measure in the limit set of dimension $\delta$. Namely, $\mu$ satisfies $\gamma^{*} \mu=\left|\gamma^{\prime}\right|^{\prime} \mu, \gamma \in \Gamma$. This was proved for discrete groups in $\mathbf{H}^{2}$ by Patterson [P] and for groups in $\mathbf{H}^{d+1}$ in [S(iii), §1].

(2) For studying the comparison of $\mu$ (ball of radius $r$ ) and $r^{\delta}$ it is useful to introduce the $\Gamma$-invariant positive eigenfunction of the Laplacian related to $\mu$.

If $\xi$ belongs to $S^{d}=\partial B^{d+1}$, let $y_{\xi}$ denote the function on $B^{d+1}$ with a pole at $\xi$ corresponding by stereographic projection to the vertical height $y$ in the upper half space. Since $y^{\delta}$ is a $\delta(\delta-d)$ eigenfunction of the hyperbolic Laplacian $\Delta$ any convex combination of the $y_{\xi}^{\delta}$ is also.

The $\mu$ convex combination of the $y_{\xi}^{\delta}$ is a positive $\delta(\delta-d)$ eigenfunction $\phi_{\mu}$ which is $\Gamma$-invariant when $\mu$ is a conformal measure, $\gamma^{*} \mu=\left|\gamma^{\prime}\right|^{\delta} \mu$. See [P] and [S(iii), §7]. The eigenfunction $\phi_{\mu}$ can be used to study the ratio $\mu$ (disk of radius $r) / r^{\delta}$. The growth of $\phi_{\mu}$ along a geodesic in $\mathbf{H}^{d+1} / \Gamma$ is always an upper bound. For certain groups including geometrically finite groups $\phi_{\mu}$ provides also a lower bound, see [S(iv), §6].

(3) From a $\Gamma$-invariant eigenfunction $\phi$ of the hyperbolic Laplacian $\Delta$ one can construct for each $p \in B^{d+1}$ a conformal measure $\mu(\phi)$ at infinity by taking a weak limit as $R \rightarrow \infty$ of the normalized measure proportional to

$$
\phi \circ(\text { measure on sphere of radius } R \text { centered at } p) \text {. }
$$

If $\delta \geqslant d / 2$, and $\phi$ is $\phi_{\mu}$, one reconstructs $\mu$ from $\phi_{\mu}$ in this way. If $\delta<d / 2$ this limit measure is not $\mu$ but like a Fourier transform of $\mu$. (This is an instance of the duality that appears in the unitary representation theory of the Moebius group - familiar for the first cases $\operatorname{PSl}(2, R)$ and $\operatorname{PSl}(2, \mathrm{C})$.)

(4) If $\Gamma$ is a geometrically finite group the conformal measure is unique and the canonical positive eigenfunction $\phi_{\mu}$ belongs to $L^{2}\left(\mathbf{H}^{d+1} / \Gamma\right)$ iff $\delta>d / 2$. If $G$ denotes the group of conformal transformations of $B^{d+1}$ then of course $\phi_{\mu}$ also belongs to $L^{2}(G / \Gamma)$. Because $\phi_{\mu}$ is an $L^{2}$ eigenfunction of the hyperbolic Laplacian $\Delta$ the $G$ orbit of $\phi_{\mu}$ generates an irreducible unitary of $G$ in $L^{2}(G / \Gamma)$. This irreducible component is the member of the complementary series labeled by $\delta$ in $(d / 2, d]$. Recalling Theorem 10 we see a geometric interpretation of the complementary series by their realization in $L^{2}(G / \Gamma)$ for discrete groups $\Gamma$ with the parameter being just the Hausdorff dimension of the limit set.

In [S(v), \$7] it was shown there is a continuous family of quasifuchsian groups where $\delta$ varied from 1 to 2 . The representations corresponding to $\phi_{\mu}$ thus cover the entire complementary series as the group $\Gamma$ varies in this deformation.

(5) For geometrically finite groups with $\delta \leqslant d / 2$ the positive eigenfunction $\phi_{\mu}$ belongs to $L^{2}$ of a part of $\mathbf{H}^{d+1} / \Gamma$ [S(iv)]. This part is the convex core defined by forming first the convex hull of the limit set in $\mathbf{H}^{d+1}$, then a unit neighborhood of this convex hull, and then the quotient by $\Gamma$. It is a problem 
whether there are any other groups besides geometrically finite groups where $\phi_{\mu}$ belongs to $L^{2}$ of $\mathbf{H}^{d+1} / \Gamma, \delta>d / 2$ (or to $L^{2}$ of the convex core $\mathbf{H}^{d+1} / \Gamma$, $\delta \leqslant d / 2)$.

Entropy. (6) The property mentioned in (5), the square integrability of the eigenfunction $\phi_{\mu}$ on the convex core of $\mathbf{H}^{d+1} / \Gamma$, implies the associated invariant measure for the geodesic flow on $\mathbf{H}^{d+1} / \Gamma$ has finite total mass [S(iv)].

In the case of finite mass we can define the measure-theoretic entropy of the geodesic flow. For geometrically finite groups one finds [S(iv)] this entropy is just the critical exponent $\delta(\Gamma)$.

If there are no cusps one can define a topological entropy for the invariant set of geodesics with both endpoints in the limit set. The topological entropy is again the critical exponent $\delta$ [S(iv), §9].

In the geometrically finite case not only is the invariant measure for the geodesic flow of finite total mass, it is also mixing and even Bernoulli [R]. This example of a geometrically defined Bernoulli flow provides a new twist on the Anosov-Pesin foliation picture producing this random phenomenon. In these examples the relevant measure class conditioned to the leaves is not the smooth measure (of dimension $d$ ) but a Hausdorff type measure of dimension $\delta$.

Hausdorff dimension and measure. (7) When as in (6) the associated measure for the geodesic flow has finite total mass (thus for all geometrically finite groups [S(iv), \$5]) the critical exponent is the Hausdorff dimension of the radial limit set $\Lambda_{r}$ of Part I [S(ii), Theorem 25]. This number then equals the Hausdorff dimension of the entire limit set for geometrically finite groups [S(iv), §2] because the difference between the radial limit set and the entire limit set is only a countable set in this case.

A corollary for general discrete groups in $\mathbf{H}^{2}$ (or general Riemann surfaces) is that the critical exponent is always the Hausdorff dimension of the radial limit set $\Lambda_{r}$. This follows because any group in $\mathbf{H}^{2}$ is a union of geometrically finite groups and $\delta$ satisfies a simple semicontinuity property for limits [S(ii), $\S 2]$.

(8) We have seen in Theorem 10 that the Hausdorff $\delta$-measure of a geometrically finite group without cusps is finite and positive (and it equals the unique conformal measure). This is sometimes true when there are cusps.

If all cusps have rank $\leqslant \delta$, the unique conformal measure is again the Hausdorff $\delta$-measure. If all cusps have rank $\geqslant \delta$ the canonical conformal measure may be described metrically by a Hausdorff packing construction dual to the customary covering definition [S(iv), §7].

If $\delta$ strictly separates the ranks of two cusps then it is a problem to give a metrical description of the unique conformal measure on the limit set of a geometrically finite group [S(iv)].

Riemannian manifolds. (9) The appearance of positive square integrable eigenfunctions above, their uniqueness coming from ergodicity, etc. prompts a more general discussion.

First of all for any complete Riemannian manifold $M$ one can define a real number $\lambda_{0} \leqslant 0$ by a sequence of equivalent conditions. 
(i) The $L^{2}$-spectrum of the Laplacian on $M$ is precisely contained in $\left(-\infty, \lambda_{0}\right]$

(ii) The infimum of the set of $\lambda$ so that for $x, y \in M \int_{1}^{\infty} e^{-\lambda t} p_{t}(x, y) d t$ converges. Here $p_{t}(x, y)$ is the heat kernel expressing the probability that a random path starting at $x$ finds itself at $y$ after time $t$.

(iii) The minimum of the set of $\lambda$ so that there is a positive eigenfunction of $\Delta$.

(10) Any positive eigenfunction $\phi_{0}$ for the smallest eigenvalue $\lambda_{0}$ is called a ground state or basic eigenfunction. There are several general results about these.

(i) Any square-integrable eigenfunction of the hyperbolic Laplacian $\Delta$ which is positive must be a ground state and belong to the eigenvalue $\lambda_{0}$.

(ii) Any square-integrable eigenfunction of $\Delta$ which belongs to $\lambda_{0}$ must be positive (or negative) and so define a ground state. (A corollary is that no two such can be orthogonal. Thus the zeroth eigenvalue $\lambda_{0}$ has $L^{2}$-multiplicity at most one.)

(iii) If there is a square-integrable ground state then any other ground state (not necessarily square integrable) must be a multiple of the first one. (The proof here and in (v) below uses the modified Markov process mentioned in Part I and [S(ii), §7].)

(iv) More generally if $\int_{1}^{\infty} e^{-\lambda_{0} t} p_{t}(x, y) d t$ diverges all ground states are multiples of any given one (not necessarily square integrable).

(11) We specialize the general Riemannian manifold to a general hyperbolic manifold $\mathbf{H}^{d+1} / \Gamma$ and connect the zeroth eigenvalue and ground states with critical exponents and $\Gamma$-invariant conformal measures. As we mentioned above conformal measures of dimension $\delta$ determine $\delta(\delta-d)$ positive eigenfunctions. Furthermore, positive eigenfunctions belonging to $\lambda<0$ determine conformal densities of dimension $\delta=d / 2+\left(\lambda+d^{2} / 4\right)^{1 / 2}$ (because $\delta(\delta-\mathrm{d})=\lambda$ and $\delta \geqslant d / 2)$.

Thus for any hyperbolic manifold $\mathbf{H}^{d+1} / \Gamma, \lambda_{0}$ is $\geqslant-d^{2} / 4$ and equality occurs iff the critical exponent $\delta(\Gamma)$ is less than or equal to $d / 2$. Otherwise, where $\delta \geqslant d / 2, \lambda_{0}$ is $\delta(\delta-d)$. For $d=1$, most if not all of this was proved by Elstrodt and Patterson.

The basic eigenfunctions or ground states correspond to the $\phi_{\mu}$ discussed above. The existence of a conformal measure for any discrete conformal group in case $\delta \geqslant d / 2$ is generalized to a statement valid for any complete Riemannian manifold-the existence of ground states.

For geometrically finite conformal groups we have a square-integrable ground state if $\delta>d / 2$. The uniqueness then following from ergodicity is thus generalized to one for complete Riemannian manifolds-the uniqueness of square-integrable ground states. For proofs see [S(vii)].

Concluding remarks. We have seen one real number $\delta(\Gamma)$ defined for any discrete conformal group $\Gamma$ acting on $B^{d+1}$ appearing in other guises for certain groups - the Hausdorff dimension of the limit set, the entropy of the geodesic flow, the "square root" of the lowest eigenvalue $\lambda_{0}$ of the Laplacian $d / 2+\left(\lambda_{0}+d^{2} / 4\right)^{1 / 2}$. 
For geometrically finite groups all these concepts mesh nicely, and these hyperbolic manifolds of infinite Riemannian volume behave after renormalization by the canonical conformal measure just like finite volume manifolds vis a vis ergodicity of the geodesic flow, square-integrable ground states, entropy and dimension, etc.

\section{BIBLIOGRAPHY}

[A] Lars Ahlfors, (i) Finitely generated Kleinian groups, Amer. J. Math. 86 (1964), 413-429.

(ii) Some remarks on Kleinian groups, Tulane Conf. Conformal mappings (1965).

(iii) Moebius transformations in several dimensions, Minnesota Book Center, Minneapolis, Minn., 1981.

[Aa] Jon Aaronson, Pointwise behaviour of infinite measure preserving transformations, Israel J. Math. Math. 32 (1979).

[As] Jon Aaronson and Dennis Sullivan, Rational ergodicity of the geodesic flow on infinite volume hyperbolic manifolds (manuscript).

[B] Lipmann Bers, (i) Spaces of Kleinian groups, Several Complex Variables, I, (Maryland, 1970).

(ii) See also these proceedings.

[B] Rufus Bowen, Hausdorff dimension of quasi-circles, Inst. Hautes Études Sci. Publ. Math.

[C et al] Alain Connes, Jack Feldman and Benjamin Weiss, Amenable equivalence relations are hyperfinite, Inst. Hautes Études Sci. Publ. Math. preprint, 1980.

[G] Lucy Garnett, Functions and measures harmonic along the leaves of a foliation, Ph.D. Thesis, Dartmouth College, 1981; Inst. Hautes Etudes Sci. Publ. Math. preprint, June 1980.

[K] Wolfgang Krieger, On ergodic flows and isomorphisms of factors, Math. Ann. 223 (1976), 19-70.

[L] William LeVeque, Continued fractions for the Gaussian field, Indag. Math. (1952).

[P] S. J. Patterson, The limit set of a Fuchsian group, Acta Math. B6 (1976), 241-273.

[R] Dan Rudolph, manuscript (Maryland).

[Rn] Marina Ratner, Rigidity of the horocycle flow, preprint, Berkeley, 1980.

[S] Dennis Sullivan, (i) Disjoint spheres, approximation by imaginary quadratic numbers and the logarithm law for geodesics, Acta Math. (to appear).

(ii) On the ergodic theory at infinity of an arbitrary discrete group of hyperbolic motions, Ann. of Math. Studies, No. 97, Princeton Univ. Press, Princeton, N.J., 1981, pp. 465-496.

(iii) The density at infinity of a discrete group of hyperbolic motions, Inst. Hautes Études Sci. Publ. Math. 50 (1979).

(iv) Entropy, Hausdorff measures old and new, and limit sets of geometrically finite Kleinian groups, Acta Math. (to appear).

(v) Growth of positive harmonic functions and Kleinian group limit sets of planar measure zero and Hausdorff dimension two, N. Kuiper 60th Birthday Volume (Holland), Springer-Verlag, 1981.

(vi) On the finiteness of cusps, Acta Math. (to appear).

(vii) The exponential size of a Riemannian manifold relative to heat flow, Acta Math. (submitted).

[Sc] Klaus Schmidt, Cocycles of ergodic group actions, Warwick Notes, 1976.

[T] Bill Thurston, (i) Geometry and topology of three-manifolds, preprint, Princeton Univ., 1978; to be published by Princeton Univ. Press, 1982.

(ii) Hyperbolic structures on 3-manifolds, I. Deformation of acylindrical manifolds, Ann. of Math. (to appear).

[Tu] Pekka Tukia, Rigidity of Kleinian groups and dimensionality of the limit set, preprint, Univ. of Helsinki, 1981.

InStitute des Haute Études Scientifique, Bures sur YvetTe, France 
